\title{
Dynamical revival of phase coherence in a many-boson system
}

\author{
Shi-Jie Yang*1 and Sumin Nie ${ }^{1}$
}

${ }^{1}$ Department of Physics, Beijing Normal University, Beijing 100875, China

\begin{abstract}
We study the quantum dynamics of cold Bose atoms in a double well. It is shown that selftrapping, as well as population oscillations are common phenomena associated to nonlinear interactions. For larger $U / t$, multi-particle tunneling is damped and the quantum dynamics is dominated by the single-particle tunneling. The many-body system can be effectively described in a truncated Fock space. It exhibits coherence-decoherence oscillations in the temporal evolution. We predict a novel phenomenon of dynamical revival and collapse of matter wave fields in optical lattices in regimes near the superfluid-Mott insulator phase boundary.
\end{abstract}

PACS numbers: 03.75.Lm, 03.75.Gg, 03.75.Kk

Tunneling through a barrier is a paradigm of quantum mechanics and usually takes place on a nanoscopic scale. The existence of long-range phase coherence, inherent to the description of a Bose-Einstein condensate (BEC) in terms of a coherent matter wave, was demonstrated experimentally [1 3]. In a simple double well, Josephson oscillations takes place in a Bose-Einstein condensate (BEC) when the initial population imbalance is below a critical value $[4]$. Above the critical value and as the repulsive interactions between the atoms become stronger, a phenomenon of macroscopic quantum self-trapping where the atoms essentially stay in one well is predicted [7, 8] and observed for the experimental lifetime [9, 10].

As to the correlated many-body system, single-particle tunneling and multi-particle tunneling take effect simultaneously and the collective motion of the atoms becomes quite complicated. Phase coherence is very difficult to establish due to the combined contributions of single- and multi-particle tunneling. Virtually, self-trapping is not the unique phenomenon of coherent matter waves, it may happen in a correlated cold atom gas under similar experimental conditions [11, 12]. The physical origin of selftrapping is, rather than the macroscopic phase coherence of the system, the nonlinear interactions. Furthermore, population oscillations between the double wells also take place, given the interaction is small and the particles are initially imbalanced. The coherent Josephson oscillations in BECs is just a special case.

In this paper, we explore the dynamical evolution of a correlated Bose gas in a double well by directly diagonalizing the Hamiltonian in the Fock space. We attempt to clarify the roles of single- and multi-particle tunneling in the quantum dynamics 13 -16]. It is shown that the so-called self-trapping, as well as the population oscillation, are natural consequences of the dynamical evolution. For larger $U / t$, the system experiences a coherence-decoherence oscillation. We further predict a phenomenon of dynamical revival and collapse of macroscopic matter waves in optical lattices, where the system parameter is near the superfluid (SF)-Mott insulator (MI) phase boundary and the state is initially in the deep Mott regimes. This remarkable phenomenon is caused by the damping of many-particle tunneling whereas the single-particle tunneling dominating the quantum dynamics.

We concern with $N$ bosonic atoms confined in symmetrical double wells (left and right). Making the two-mode approximation and considering only the lowest energy band, the creation and annihilation operators $\left(\hat{a}_{1,2}^{\dagger}\right.$ and $\hat{a}_{1,2}$ respectively) for atoms localized in either side of the well can be constructed. The Bose-Hubbard Hamiltonian is written as 1,17 ]

$$
\hat{H}=-t\left(\hat{a}_{1}^{\dagger} \hat{a}_{2}+\hat{a}_{2}^{\dagger} \hat{a}_{1}\right)+\frac{U}{2}\left[\hat{n}_{1}\left(\hat{n}_{1}-1\right)+\hat{n}_{2}\left(\hat{n}_{2}-1\right)\right],
$$

where $U$ is the repulsion between a pair of bosons occupying the same site, $t$ is the hopping coefficient, and $\hat{n}_{i}(i=1,2)$ are the number operators. Consistence of this model requires $\frac{1}{2} U n_{i}\left(n_{i}-1\right) \ll \hbar \widetilde{\omega}_{i}$, where $\widetilde{\omega}_{i}$ is the characteristic frequency of the well in the harmonic approximation.

The Hamiltonian (11) is explicitly represented in the Fock basis set $\{|N, 0\rangle,|N-1,1\rangle, \cdots,|0, N\rangle\}$ as

$H=\left(\begin{array}{cccc}\frac{U}{2}\left(N^{2}-N\right) & -t \sqrt{N} & \cdots & 0 \\ -t \sqrt{N} & \frac{U}{2}\left(N^{2}-3 N+2\right) & \cdots & 0 \\ \vdots & \vdots & \ddots & \vdots \\ 0 & 0 & \cdots & \frac{U}{2}\left(N^{2}-N\right)\end{array}\right)$

The general eigenstates are expressed as linear combinations of the occupation bases, $\left|\psi_{j}\right\rangle=\sum_{k=0}^{N} c_{j k}|N-k, k\rangle$ $(j=0,1, \cdots, N)$, which correspond to the eigenvalues $\omega_{j}$. The coefficients $c_{j k}$ satisfy the recursive relation 
*Corresponding author: yangshijie@tsinghua.org.cn

$$
-t \sqrt{(N-k)(k+1)} c_{j(k+1)}-t \sqrt{(N-k+1) k} c_{j(k-1)}+\left[\frac{U}{2}\left(N^{2}-2 N k-N+2 k^{2}\right)-\omega_{j}\right] c_{j k}=0 .
$$

The temporal evolution of the state is governed by the

Schrödinger equation for a given initial state $|\Psi(0)\rangle$.

$$
|\Psi(\tau)\rangle=\sum_{j=0}^{N} f_{j}(\tau)\left|\psi_{j}\right\rangle=\sum_{k=0}^{N}\left(\sum_{j=0}^{N} f_{j}(0) c_{j k} e^{-i \omega_{j} \tau}\right)|N-k, k\rangle \equiv \sum_{k=0}^{N} g_{k}(\tau)|N-k, k\rangle,
$$

with $f_{j}(0)=\left\langle\psi_{j} \mid \Psi(0)\right\rangle$ and $g_{k}(\tau)=$ $\sum_{j=0}^{N} f_{j}(0) c_{j k} e^{-i \omega_{j} \tau}$.

The population imbalance between the two wells is evaluated by $Z(\tau) \equiv\left\langle\Psi\left|\left(\hat{n}_{1}-\hat{n}_{2}\right)\right| \Psi\right\rangle / N=\sum_{k=0}^{N}(1-$ $2 k / N)\left|g_{k}(\tau)\right|^{2}$. To depict the coherence degree of the system, we introduce a characteristic parameter

$$
\alpha(\tau)=\frac{\left|\lambda_{1}-\lambda_{2}\right|}{\lambda_{1}+\lambda_{2}},
$$

where $\lambda_{1}$ and $\lambda_{2}$ are the two eigenvalues of the singleparticle density $\rho_{\mu \nu}(\tau)=\left\langle\Psi(\tau)\left|\hat{a}_{\mu}^{\dagger} \hat{a}_{\nu}\right| \Psi(\tau)\right\rangle(\mu, \nu=$ $1,2)$ [18, 19]. When $\alpha \rightarrow 1$, the system is in the coherent (quasicoherent) state since in this case there is only one large eigenvalue of matrix $\rho_{\mu \nu}(\tau)$. Accordingly, $\alpha \rightarrow 0$ indicates the system is in the decoherent or fragmented state because there are two densely populated natural orbits. One can also use the quantum fluctuations of the particle number in one of the wells, $\sigma_{1}(\tau)=\sqrt{\left\langle\left[\hat{n}_{1}(\tau)-\left\langle\hat{n}_{1}(\tau)\right\rangle\right]^{2}\right\rangle} /\left\langle\hat{n}_{1}(\tau)\right\rangle$, to describe the coherence of the system [21]. In the weak interaction, strong tunneling limit $(U / t \ll 1)$, each atom is in a coherent superposition of left-well and right-well states. The ground state of the system is a state with a mean number $N / 2$ of atoms in each well with Poissonian fluctuations $\sigma_{1}=\sqrt{N / 2}$. The states in each well are quasicoherent states as the total number of atoms in the system is fixed. In the opposite limit of strong interactions or weak tunneling $(U / t \gg 1)$, the tunneling term is negligible. In this case, the Hamiltonian is the product of number operators for the left and right wells. The eigenstates are products of Fock states $\left(\sigma_{1}=0\right)$ and are referred as decoherent states. This regime is analogous to the MI phase in a lattice system.

In the following we consider two typical cases in which the initial state is a coherent state or a Fock state. We choose $N=10$ and set $t=1$ as the units.

Initial coherent states We first study the quantum dynamics of an $N$-particle system which is initially in a coherent state,

$$
|\Psi(0)\rangle=\left(\frac{\hat{a}_{1}^{\dagger}+\hat{a}_{2}^{\dagger}}{\sqrt{2}}\right)^{N}|0\rangle .
$$

The particles are evenly distributed in the two wells, i.e., $Z(\tau) \equiv 0$, whereas the number fluctuations persist. Figure 1 shows the temporal evolution of parameters $\alpha$ and $\sigma_{1}$ for various interactions $U / t$. When $U / t$ is small, $\alpha(\tau)$ fluctuates around unit and the system preserves coherence or quasicoherence. This is analogous to the SF in optical lattices. As $U / t$ increases, the value of $\alpha$ lowers, indicating the coherence is gradually destroyed. However, when $U / t$ further increases, $\alpha(\tau)$ again exhibits a nearly periodic oscillation. In the limit of $U / t \gg 1$, the value of $\alpha$ oscillates between unit and zero with a period of $T=\pi / U$, implying the system experiences revivals and collapses of the coherence.

This phenomenon can be explained as follows. The initial state can be expanded as $|\Psi(0)\rangle \sim$ $\sum_{k=0}^{N} C_{N}^{k}\left(\hat{a}_{1}^{\dagger}\right)^{N-k}\left(\hat{a}_{2}^{\dagger}\right)^{k}|0\rangle$. When $U / t \gg 1$, the Fock states $\left(a_{j}^{\dagger}\right)^{k}|0\rangle$ in each well are the eigenstates and the corresponding eigenenergies are $U k(k-1) / 2$. The system is then a superposition of products of the Fock states, which evolves independently as

$$
|\Psi(\tau)\rangle \sim \sum_{k=0}^{N} C_{N}^{k} e^{-\frac{i}{2} U(N-k)(N-k-1) \tau}\left(\hat{a}_{1}^{\dagger}\right)^{N-k} e^{-\frac{i}{2} U k(k-1) \tau}\left(\hat{a}_{2}^{\dagger}\right)^{k}|0\rangle
$$



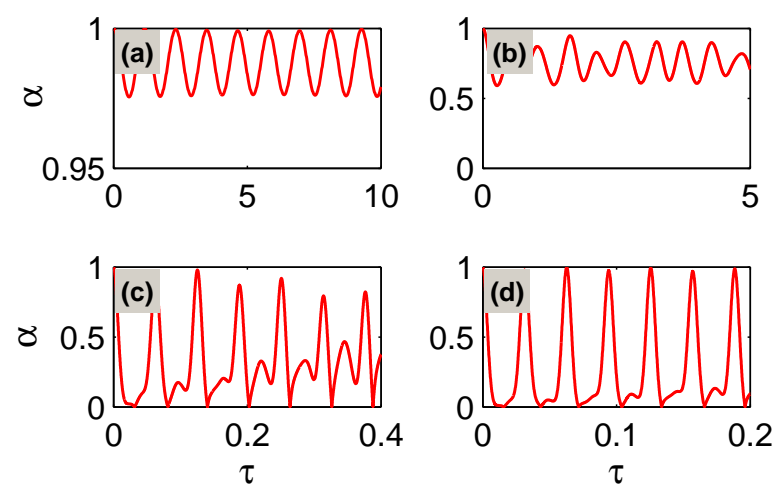

FIG. 1: (Color online) Temporal evolution of $\alpha(\tau)$ for the $N=10$ system initiating from the coherent state. From (a) to (d), $U / t=0.2,2,50,100$, respectively. Periodic revivals of coherence is clearly enhanced for $U / t \gg 1$.

$$
=e^{-\frac{i}{2} U N(N-1) \tau} \sum_{k=0}^{N} C_{N}^{k} e^{-\frac{i}{2} U(N-k) k \tau}\left(\hat{a}_{1}^{\dagger}\right)^{N-k}\left(\hat{a}_{2}^{\dagger}\right)^{k}|0\rangle .
$$
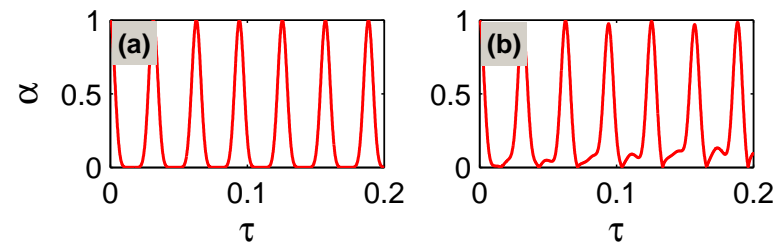

FIG. 2: (Color online) Comparison of evolution of $\alpha(\tau)$ between (a) independent and (b) full quantum dynamics at $U / t=100$. The coherence-decoherence oscillation period is $T=\pi / U$.

There attaches a time-dependent phase factor in each term. When the time evolves an integer multiples of $\pi / U$, the system recovers its initial coherent state. In between this period, superposition from various terms in (8) cancels and the coherence is destroyed. Figure 2 displays the coincidence of the full and the independent evolving dynamics.

This result has the same gradients of the experiment carried out by Greiner et al, where they studied the dynamical evolution of the matter wave field in a 3dimensional optical lattice 20]. While the system is initially prepared in a superfluid state, the experimental parameters are suddenly changed to the deep Mott regimes. A periodical collapse and revival of the matter wave fields were found in the temporal evolution.

Initial Fock states We now explore our main results for the many-body system initially in a symmetrical Fock state,

$$
|\Psi(0)\rangle=|N / 2, N / 2\rangle
$$

It is found that for all values of $U / t, Z(\tau) \equiv 0$, which implies the atoms are evenly distributed in the two wells. But the quantum fluctuations of the particle population in each well persist. Figure 3 shows the coherence degree $\alpha(\tau)$ (solid lines) and the number fluctuations $\sigma_{1}(\tau)$ (dot lines) almost evolve synchronically. Intriguingly, with the enhancement of interaction, the system gradually exhibits a regular oscillating feature [Fig.3(c),(d)].

This remarkable phenomenon origins from the damping of the multi-particle tunneling due to the constraint of energy conservation. According to the quantum perturbation theory, the hopping coefficient for single particle tunneling is $t$. For two-particle co-tunneling it becomes $t^{2} / U$. As $U \gg t$, the single-particle tunneling process will dominate the quantum dynamics. With this consideration, the Hamiltonian can be effectively approximated in a truncated Fock space by omitting the multi-particle tunneling, $\{|N / 2-1, N / 2+1\rangle,|N / 2, N / 2\rangle, \mid N / 2+1, N / 2-$ $1\rangle\}$, where only the single-particle tunneling is relevant. 


$$
\tilde{H}=\left(\begin{array}{ccc}
U\left[(N / 2)^{2}-N / 2+1\right] & -t \sqrt{N / 2(N / 2+1)} & 0 \\
-t \sqrt{N / 2(N / 2+1)} & U\left[(N / 2)^{2}-N / 2\right] & -t \sqrt{N / 2(N / 2+1)} \\
0 & -t \sqrt{N / 2(N / 2+1)} & U\left[(N / 2)^{2}-N / 2+1\right]
\end{array}\right)
$$
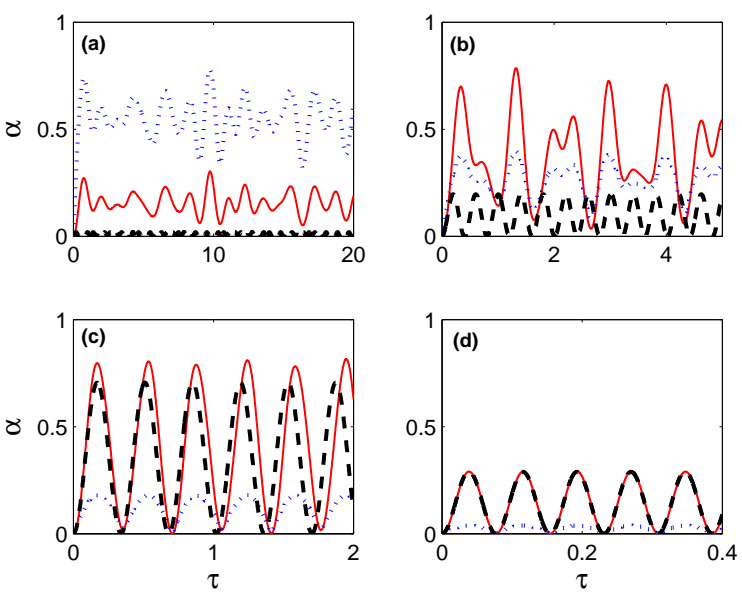

FIG. 3: (Color online) Quantum dynamics for the $N=10$ system with the initial Fock state $|\Psi(0)\rangle=|5,5\rangle$. The solid lines describe $\alpha(\tau)$ and the dotted lines describe the particle number fluctuations $\sigma_{1}(\tau)$ in one of the double well. From (a) to $(\mathrm{d}), U / t=0.2,2,10,80$. The broken curves are $\alpha(\tau)$ calculated in the truncated Fock space.

The broken curves in Fig.3 depict the temporal evolution of $\alpha(\tau)$ calculated with $\tilde{H}$ in the truncated Fock space. The coincidence with the curves in the full Fock space (Fig.3(c) and (d)) verify our explanation. Further increasing the interaction will suppress the coherence (Fig.3(d)) as the single-particle tunneling is also damped. It can be proven from (9) that in the limit of $U / t \gg 1, \alpha(\tau)$ oscillates sinusoidally with a period of $T \sim 2 \pi / U$.

Based on our result in the double well system, we predict a novel phenomenon of dynamical revivals of macroscopic matter waves for cold Bose atoms in optical lattices by comparison to the experiment by Greiner et al [20]. Suppose the system is initially in the deep Mott regimes, one suddenly changes the experimental parameters to regimes near the SF-MI phase boundary, where only the single-particle tunneling dominates. We can observe creation and destruction of macroscopic phase coherence in the temporal evolution. At first sight, our prediction seems the reverse process of that of Greiners'. The underlying mechanism is quite different. In our case, macroscopic phase coherence is dynamically generated from a MI and the single-particle tunneling plays a key role. Most notably, if the system parameters are directly changed to deep SF regimes $(U / t \ll 1)$, where the multiparticle tunneling becomes as important as the singleparticle tunneling, no macroscopic phase coherence will occur. Orzel et al[21] had suggested the possibility of observing the temporal evolution of SF order parameter. Basically, the bosons are prepared in the number squeezed Mott state, and then the potential is suddenly reduced into the superfluid phase. The consequent evolution of the superfluid order can be deduced from the intensity of interference patterns appearing when the atoms are released from the trap at sequential times. In a recent experiment, S. Will et al 22] observed multi-body interactions in the time-resolved coherent quantum phase revivals.

Altman et al 23] have studied the dynamics of bosons in an optical lattice using a modified coherent states path integral. They suggested that a system prepared in the unstable Mott state is expected to exhibit macroscopic oscillations of the superfluid order parameter. Our work explicitly clarified the roles of multi- and single-particle tunneling. We point out that the revival of coherence takes place only near the phase transition where the multi-particle tunneling is damped. If the system is directly changed to deep superfluid regimes, then no revival of the coherence will be observed. This result is somehow contradict to one's intuition and is critical for experimental observation.

Finally, we briefly discuss the system evolves from an asymmetrical Fock state, e.g., $|\Psi(0)\rangle=|10,0\rangle$. Figure 4 shows the evolution of the population imbalance $Z(\tau)$. In the weak interaction regimes (Fig.4(a)), $Z(\tau)$ varies between -1 to 1 , indicating a phenomenon analogous to the Josephson oscillation in BEC. We emphasis the population oscillations is not the Josephson effect since the system is not in a macroscopic coherent state. As the interaction increases, $Z(\tau)$ remains positive. Most of the particles keep staying in one of the wells due to the constraint of energy conservation. This is the so-called self-trapping phenomenon. It is notable that in this case there is no revival of phase coherence.

In summary, we have investigated the dynamical evolution of cold Bose atoms in a double well. We conclude that self-trapping and population oscillations between the two wells are consequences of nonlinear interactions and energy conservation instead of the macroscopic phase coherence. We predict a novel phenomenon of revivals and collapses of macroscopic phase coherence from deep MI regimes in optical lattices. Our results may be 

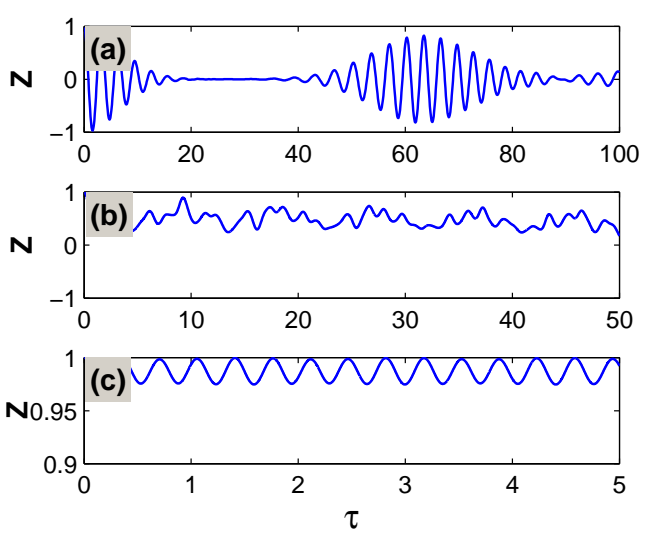

FIG. 4: (Color online) Temporal evolution of the population imbalance $Z(\tau)$ for $N=10$ atoms starting from the Fock state $|10,0\rangle$. From (a) to (c), the interaction $U / t=0.1,0.5,2$. Self-trapping occurs when $U / t \gtrsim 2$.

helpful to understand how a macroscopic phase coherence can dynamically be generated from an incoherent state and the roles of particle tunneling in the dynamical evolution of quantum many-body system.

We thank M. Ueda and S. Will for helpful discussions. This work is supported by the National Natural Science Foundation of China under grant No. 10874018, the 973 Program Project under grant No. 2009CB929101 and "the Fundamental Research Funds for the Central Universities".

[1] D. Jaksch, C. Bruder, J.I. Cirac, C.W. Gardiner, and P. Zoller, Phys. Rev. Lett. 81, 3108(1998).

[2] S. Stringari, C.R. Acad. Sci. 4, 381(2001).
[3] M. Greiner, O. Mandel, T. Esslinger, T.W. Hänsch, and I. Bloch, Nature 415, 39(2002).

[4] J. Javanainen, Phys. Rev. Lett. 57, 3164 (1986).

[5] M.W. Jack, M. J. Collett, and D. F.Walls, Phys. Rev. A 54, R4625 (1996).

[6] I. Zapata, F. Sols, and A. J. Leggett, Phys. Rev. A 57, R28 (1998).

[7] A. Smerzi, S. Fantoni, S. Giovanazzi, and S. R. Shenoy, Phys. Rev. Lett. 79, 4950 (1997).

[8] S. Raghavan, A. Smerzi, S. Fantoni, and S. R. Shenoy, Phys. Rev. A 59, 620 (1999).

[9] Th. Anker, M. Albiez, R. Gati, S. Hunsmann, B. Eiermann, A. Trombettoni, and M. K. Oberthaler, Phys. Rev. Lett. 94, 020403(2005).

[10] M. Albiez, R. Gati, J. Fölling, S. Hunsmann, M. Cristiani, and M.K. Oberthaler, Phys. Rev. Lett. 95, 010402(2005).

[11] C.E. Creffield, Phys. Rev. A 75, 031607(R)(2007).

[12] G. J. Milburn, J. Corney, E. M. Wright, and D. F. Walls, Phys. Rev. A 55, 4318(1997).

[13] K. Winkler, G. Thalhammer, F. Lang, R. Grimm, J. Hecker Denschlag, A. J. Daley, A. Kantian, H. P. Büchler, and P. Zoller, nature 441, 853(2006).

[14] S. Fölling, S. Trotzky, P. Cheinet, M. Feld, R. Saers, A. Widera, T.Müller, and I. Bloch, Nature 448, 1029(2007).

[15] S. Zöllner, H. Meyer, and P. Schmelcher, Phys. Rev. Lett. 100, 040401(2008).

[16] U.R. Fischer and R. Schützhold, Phys. Rev. A 78, 061603(R)(2008).

[17] A. Vardi and J.R. Anglin, Phys. Rev. Lett. 86, 568(2001).

[18] O. Penrose and L. Onsager, Phys. Rev. 104, 576(1956).

[19] E.J. Mueller, T.-L. Ho, M. Ueda, and G. Baym, Phys. Rev. A 74, 033612(2006).

[20] M. Greiner, O. Mandel, T.W. Hänsch, and I. Bloch, Nature $419,51(2002)$.

[21] C. Orzel, A. K. Tuchman, M. L. Fenselau, M. Yasuda, and M. A. Kasevich, Science 291, 2386(2001);

[22] S. Will, T. Best, U. Schneider, L. Hackermüller, D.-S. Lühmann, and I. Bloch, Nature 465, 197(2010).

[23] E. Altman and A. Auerbach, Phys. Rev. Lett. 89, 250404(2002). 